\title{
DUMMY VARIABLE REGRESSION MODELS AND ANALYSIS OF RESPONSES IN DIAGNOSTIC SCREENING TESTS WITH REPLICATIONS
}

\author{
I. C.A. Oyeka and E. U. Umeh \\ Department of Statistics, Nnamdi Azikiwe University, Awka \\ editus2002@yahoo.com
}

\section{INTRODUCTION}

Often in controlled comparative either prospective, retrospective or cross-sectional study involving matched samples of subjects or patients, the response of a subject or patient to a predisposing factor in a retrospective study or to a condition or treatment in a prospective study may be dichotomous. But there may also be several other possible response options other than dichotomous but numbering some $\mathrm{c}(c \geq 2)$ possible mutually exclusive response options or categories. Specifically, suppose we have a total random sample of $n$ - matched pairs of patients or subjects matched on certain characteristics to be exposed to two treatments, drugs, tests; suppose $n_{i j}$ pairs of these subject pairs are in the $i^{\text {th }}$ response category by case and $j^{\text {th }}$ response category by the control subject, for $i, j=1,2, \ldots, c$ responses. There may be no replications; that is there is only one pair of case-control subjects.

\section{PROPOSED METHOD}

The proposed method assumes that there are replications; that is there is more than one pair of case-control subjects in various case-control subject treatment combinations. Also, there assumed that there are no interactions between combinations or that such interaction has been removed by appropriated data transformation. This is an alternative method of analysis in terms of probabilities and odds of occurrence of outcomes using dummy variable regression techniques

\section{CONCLUSION}

The fitted regression model was obtained which explained the total variation in case and control subjects in their response scores, suggesting that its regression coefficients are all probably not different from zero. With this conclusion, further analysis would ordinarily become unnecessary, however, for illustrative purposes only, one could assume that not all the regression coefficients are zero thereby enabling some comparisons of these parameters if so desired. 